BULl. AUSTRAL. MATH. SOC.

VOL. 33 (1986), 161-175.

\title{
LAMBEK'S OPERATIONAL CATEGORIES
}

\author{
C, B, JAY
}

\begin{abstract}
An operational category is a category of models for an equational theory where the interpretation of some operations is predetermined. Examples include the equational and co-equational categories of Linton, categories of functors preserving some class of limits, and algebras for a prop as defined by Maclane. The chief result is a characterisation of the operational categories and functors in terms of their internal structure.
\end{abstract}

\section{0 . Introduction}

The class of operational categories includes those of Lawvere's algebraic categories [8] and the equational categories of Linton [9],[10], which include the tripleable categories. Since the dual of an operational category is one too, coequational and cotripleable categories are operational. The definition of operational category used here is that introduced by $\mathrm{J}$. Lambek in his lecture to the Midwest Category Theory Seminar at Waterloo University in 1968, as distinct from that of o. Wyler in [15].

Received 13 May 1985. Research supported by the Department of Mathematics and Statistics, McGill University, Montreal, and the Association of Universities and Colleges of Canada.

Copyright Clearance Centre, Inc. Serial-fee code: 0004-9727/86 $\$ \mathrm{~A} 2.00+0.00$. 
This paper is primarily a summary of [6], though there are new examples and the notation has been altered. Other generalisations of equational categories may be found in [3], [4], [14] and [15].

Our chief purpose here is to characterise the operational categories over some fixed category $A$. This done by constructing a tower of triples $S_{n}$ based on Cat/A with a limit $S_{\star}$-Alg whose image in Cat/A consists of exactly the operational categories and operational functors. In the process it is proved that there is a fixed, finite theory, from which every category of operational algebras can be constructed. This allows it to be shown that categories of algebras for props (MacLane [11]) are also operational.

I would like to thank Professor J. Lambek for introducing me to operational categories and the problem of their characterisation, and also Professor G. M. Kelly for showing me how to present mathematics clearly, yet concisely.

\section{Operational categories}

Let $U$ be a Grothendieck universe. All categories used here will have their set of morphisms in $U$ and the category of these is denoted Cat . Fix a category $A$. In some examples there is an element $V$ of $U$ which is also a Grothendieck universe with $A$ being the category of $V$-small sets.

A presentation, $(\theta, H)$, consists of a theory $\theta: B \rightarrow T$, by which is meant a functor that is bijective on objects, and a functor $H: A \times B \rightarrow C$ called the base functor. A morphism of presentations $(j, k):(\theta, H) \rightarrow\left(\theta^{\prime}, H^{\prime}\right)$ consists of $j=\left(j_{1}, j_{2}\right)$ where $j_{1}: B^{\prime} \rightarrow B$ and $j_{2}: T^{\prime} \rightarrow T$ are functors such that $\theta j_{1}=j_{2} \theta^{\prime}$, and a functor $k: \mathcal{C} \rightarrow \mathcal{C}^{\prime}$ such that $K H\left(1 \times j_{1}\right)=H^{\prime}$. Together they form a category $\operatorname{Pres}(A)$.

We define as follows a functor $O p(A): \operatorname{Pres}(A) \rightarrow$ Cat $/ A$ : it sends a presentation $(\theta, H)$ to $U: D \rightarrow A$ given by the pullback (1.1) where $H^{*}$ corresponds to $H$, and sends a morphism of presentations to the induced functor between the pullbacks. An object $U: D \rightarrow A$ of Cat/A, identified loosely with the category $D$, is called a category of operational algebras if it is the image under $0 p(A)$ of some presentation, 
(1.1)

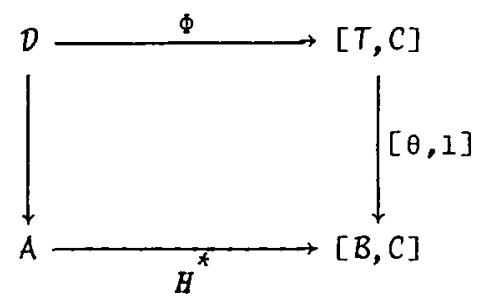

and it is called an operational category if it is equivalent in Cat/A to some category of operational algebras. A morphism in $\mathrm{Cat} / \mathrm{A}$ is an operational functor if it is equivalent to a functor in the image of $0 p(A)$. Note that $O p(A)$ is neither full nor faithful.

$B$ may be thought of as a language, the objects being sorts and the morphisms operations, into which new operations and equations may be added via $\theta$. Then $H^{*}$ maps each object of $A$ to an interpretation of $B$ in $C$, and the algebras extend these interpretations of the language to models of the theory.

For notational convenience, if $\omega$ is a morphism of $T$, that is an operation, then $\Phi_{D} \omega$ may be written as $\omega_{D}$.

EXAMPLE 1.1. Let $N$ be a skeleton of the category of finite sets so that $N^{O P}$ is the language of (one-sorted) finite products and let $H:$ Set $\times N^{O P} \rightarrow$ Set be the restriction of the homfunctor. Consider a category $T$ with finite products and a theory $\theta$ lying in $\left[N^{O P}, T\right]_{P}$ (the category of product-preserving functors from $N^{O P}$ to $T$ ). Then the resulting operational category is the category of algebras for the Lawvere theory, namely $[T, \text { Set }]_{p}$.

EXAMPLE 1.2. Let $S$ be the category of $V$-small sets for some universe $V$ in $U$. If $A$ is an S-category, with homfunctor $H: A \times A^{O P} \rightarrow S$ and $\theta \in\left[A^{O P}, T\right]_{p}$ then the resulting category of algebras is equational in Linton's sense.

EXAMPLE 1.3. Given any natural transformation $\alpha: F \Rightarrow G: A \rightarrow B$, let $\operatorname{Inv}(\alpha) \rightarrow A$ be the full subcategory of $A$ of objects $A$ for which $\alpha_{A}$ is an isomorphism. Then $\operatorname{Inv}(\alpha)+A$ is operational. The theory is the inclusion of the arrow category $\$$ into the chaotic category on two 
elements. The base functor is $H: A \times 2 \rightarrow B$ with $H^{\star} A=\alpha_{A}$. For example, if $\alpha$ is the unit for the reflection from presheaves to sheaves on some site then Inv( $\alpha)$ is just the sheaves. Also, given a limit (or a family of limits) in $K$ and a complete category $L$ then the functors from $K$ to $L$ which preserve the limit (or limits) form a full operational subcategory of $[K, L]$.

LEMMA 1.4. If $U: D \rightarrow A$ is a category of operational algebras then $U$ is faithful, reflects isomorphisms and creates coequalizers of U-split pairs.

Proof. Let $D$ have a presentation $(\theta, H)$. Since $\theta$ is bijective on objects, $[\theta, 1]$ has the desired properties, and these are preserved by pullbacks.

LEMMA 1.5. Let $U: D \rightarrow A$ be a category of operational algebras. Then $U^{O P}: D^{O P} \rightarrow A^{O P}$ and $[Y, U]:[Y, D] \rightarrow[Y, A]$ as well as the pullback of $D$ by any $A^{\prime} \rightarrow A$ are all categories of operational algebras.

Proof. $\theta^{O P}$ and $Y \times \theta$ as well as $\theta$ are all bijective on objects.

Lemma 1.4 together with Beck's Tripleability Theorem ([12]) yields the following result.

THEOREM 1.6 (Lambek). $U: D \rightarrow A$ is tripleable if and only if $U$ is operational and has a left adjoint.

Conversely, if $D$ has and $U$ preserves pullbacks then $U$ is operational if it creates coequalizers of $U$-split coequalisers (see [6]).

\section{Operational retracts}

Let $(\theta, H)$ be a presentation and $U: D \rightarrow A$ the resulting category of operational algebras. Consider a diagram in $A$ of the following type:

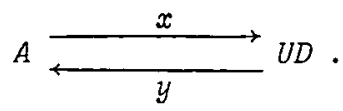

For each morphism $\omega$ in $T$ define $\phi \omega$ by 


$$
\phi \omega=H(y, 1) \omega_{D} H(x, 1) .
$$

LEMMA 2.1. $\phi$ is a functor from $T$ to $C$ if and only if $H(y x, 1)=1$ and for each composite $\omega^{\prime} \omega$ of morphisms in $T$

$$
H(y, 1) \cdot \omega_{D}^{\prime} \cdot H(x y, 1) \cdot \omega_{D} \cdot H(x, 1)=H(y, 1)\left(\omega^{\prime} \omega\right) D^{H(x, 1)} .
$$

Further, if $\phi$ is a functor then $(A, \phi)$ is an algebra.

Proof. $H(y x, 1)=1$ if and only if $\phi$ preserves identities and $\phi$ satisfies (2.3) if and only if it preserves composites. For the second statement note that if $H(y x, 1)=1$ then $\phi \theta=H(A,-)$.

In the light of the above proposition we define a diagram $(A, y, D, x)$ as in (2.1) to be an operational retract if $H(y x, 1)=1$ and (2.3) holds. Then (2.2) defines an algebra structure for $A$.

EXAMPLE 2.2. Let $U: D \rightarrow A$ be a category of operational algebras. Given a $U$-split coequalizer diagram in $A$

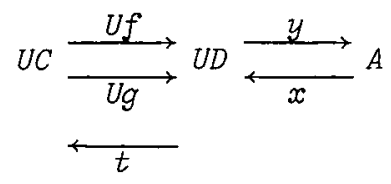

with

$$
\begin{aligned}
y \cdot U f & =y \cdot U g \\
y x & =1 \\
U f \cdot t & =1 \\
U g \cdot t & =x y
\end{aligned}
$$

then Lemma 1.4 implies that $(A, y, D, x)$ is an operational retract. (It is instructive to prove (2.3) directly from (2.4).)

LEMMA 2.3. Let $U: D \rightarrow A$ be a category of operational algebras and $(A, y, D, x)$ be on operational retract determining the algebra $(A, \phi)$. Then

(i) If $y=y^{\prime}$. Uf for some $f: D \rightarrow D^{\prime}$ then $\left(A, y^{\prime}, D^{\prime}, x^{\prime}\right.$ ) (with $\left.x^{\prime}=U f . x\right)$ is another operational retract determining $(A, \phi)$.

(ii) If $D$ is the algebra determined by some operational retract $\left(A^{\prime}, y^{\prime}, D^{\prime}, x^{\prime}\right)$ then $\left(A, y y^{\prime}, D^{\prime}, x^{\prime} x\right)$ is another operational retract determining $(A, \phi)$. 
Proof. For (i) note that the components of the natural transformation $\Phi_{D} f$ are given by $H(U f, I)$ and so

$$
\begin{aligned}
H(y, 1) \cdot \omega_{D} . H(x, 1) & =H\left(y^{\prime}, 1\right) \cdot{ }_{D} f . \Phi_{D} \omega \cdot H(x, 1) \\
& =H\left(y^{\prime}, 1\right) \cdot{ }_{D^{\prime}} \cdot H\left(x^{\prime}, 1\right) .
\end{aligned}
$$

The proof of (ii) is trivial.

Given an arbitrary functor $U: D \rightarrow A$, then there are some retracts, namely the shuffle retracts defined below, which are forced to be operational retracts if $D$ is an operational category.

Let $U: D \rightarrow A$ be an object of Cat/A and let $\equiv$ be the smallest equivalence relation on diagrams in $A$ of the form

$$
A \stackrel{x}{\longrightarrow} U D \stackrel{y}{\longrightarrow} A^{\prime}
$$

such that $(y U f, D, x) \equiv\left(y, D^{\prime}, U f . x\right)$ where $f: D \rightarrow D^{*}$ in $D$. This is called a right shuffle of $f$ over $D$. (The equivalence classes may be thought of as the connected components of an appropriate category of functors.) Similarly let $\equiv$ be the smallest equivalence relation on diagrams in $A$ of the form

$$
A \stackrel{x}{\longrightarrow} U C \stackrel{z}{\longrightarrow} U D \stackrel{y}{\longrightarrow} A^{\prime}
$$

$\left(z \neq U z^{\prime}\right.$ in general) such that if $(z, D, x) \equiv\left(z^{\prime}, D^{\prime}, x^{\prime}\right)$ then $(y, C, z, D, x) \equiv\left(y, C, z^{\prime}, D^{\prime}, x^{\prime}\right)$ and if $(y, C, z) \equiv\left(y^{\prime}, C^{\prime}, z^{\prime}\right)$ then $(y, C, z, D, x) \equiv\left(y^{\prime}, C^{\prime}, z^{\prime}, D, x\right)$ (here shuffing occurs over $C$ and $D$ ). The (1-)shuffle retracts are those retracts $(y, D, x)$ such that $(y, D, x y, D, x) \equiv(y, D, 1, D, x)$. The domain of $x$ is called the domain of the retract. A (1-) shuffle morphism between shuffle retracts from $(y, D, x)$ to $\left(y^{\prime}, D^{\prime}, x^{\prime}\right)$ is a morphism $f: \operatorname{dom} x \rightarrow \operatorname{dom} x^{\prime}$ such that $(f y, D, x) \equiv\left(y^{\prime}, D^{\prime}, x^{\prime} f\right)$.

Define $S_{1}:$ Cat $/ \mathrm{A} \rightarrow \mathrm{Cat} / \mathrm{A}$ as follows: $S_{1} D$ is the category of shuffle retracts and shuffle morphisms with $S_{1} U: S_{1} D \rightarrow A$ sending shuffle retracts to their domains and shuffle morphisms to themselves as morphisms of $A$. Given $G: D \rightarrow D^{\prime}$ in Cat $/ A, S_{1} G(y, D, x)=(y, G D, x)$ and $S_{1} G f=f \cdot S_{1}$ underlies a triple $\left(S_{1}, n, \mu\right)$ with unit $n_{D}: D \rightarrow S_{1} D$ and multiplication $\mu_{D}: S_{1}^{2} D \rightarrow S_{1} D$ given by 


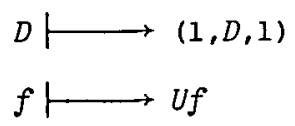

and

$$
\begin{aligned}
\left(y^{\prime},(y, D, x), x^{\prime}\right) & \longmapsto\left(y^{\prime} y, D, x x^{\prime}\right) \\
f & \longmapsto f
\end{aligned}
$$

respectively. The proof that the triple is well-defined may be found in [6]. A typical $s_{1}$-algebra will be denoted $\left(0, d_{0}, d_{1}\right)$ where $d_{0}=U: D \rightarrow A$ and $d_{1}: S_{1} D \rightarrow D$ is the structure map.

If $D$ is operational then the shuffle retracts are operational retracts and $D$ is an $S_{1}$-algebra. Not all $S_{1}$-algebras are operational, however: let $\left(D, d_{0}, d_{1}\right)$ be an $S_{1}$-algebra such that $(y, D, x)$ and $\left(y^{\prime}, D^{\prime}, x^{\prime}\right)$ are shuffle retracts with $d_{1}\left(y^{\prime}, D^{\prime}, x^{\prime}\right)=D$. Then in general it does not follow that $\left(y y^{\prime}, D^{\prime}, x^{\prime} x\right)$ is a shuffle retract, and so by Lemma 2.3(ii) not all operational retracts are 1 -shuffle retracts. In an effort to obtain this property we enlarge our equivalence relations.

Given an $S_{1}$-algebra $\left(0, d_{0}, d_{1}\right)$, let $\equiv_{2}$ be the smallest equivalence relation containing $\equiv$ such that if $(y, D, x)$ is a shuffle retract with $d_{1}\left(y^{\prime}, D^{\prime}, x^{\prime}\right)=D$ then $\left(y y^{\prime}, D^{\prime}, x^{\prime} x\right) \equiv_{2}(y, D, x)$. The definition of $\equiv_{2}$ is the same as that of $\equiv$ with $\equiv$ replaced by $\equiv_{2}$. By replacing $\equiv$ and $\equiv$ by $\equiv_{2}$ and $\equiv_{2}$ respectively, we obtain definitions of 2-shuffle retract, 2-shuffle morphism and of a triple $S_{2}$ on $S_{1}$-Alg. The $S_{2}$-algebras are not all operational either since the closure problem revealed by Lemma $2.3(i i)$ remains. Iteration of this process yields for each $n$ a triple $S_{n+1}$ on $S_{n}$-Alg for all finite $n$.

Let $S_{*}$-Alg be the limit of the $S_{n}$-Alg with their forgetful functors $U_{n}: S_{n}-\mathrm{Alg} \rightarrow S_{n-1}-\mathrm{Alg}$ (and $S_{0}-\mathrm{Alg}=\mathrm{Cat} / \mathrm{A}$ ). Then the objects of $S_{*}-\mathrm{Alg}$ are $\left(D,\left\{d_{n}\right\}\right)$ where, for each $n,\left(D, d_{0}, d_{1}, \ldots, d_{n}\right)$ is an $S_{n}$-algebra and the morphisms are those functors which are $S_{n}-$ homomorphisms for each $n$. The induced forgetful functor 
$U_{\star}: S_{\star}-\mathrm{Alg} \rightarrow \mathrm{Cat} / \mathrm{A}$ has a left adjoint $F_{\star}$ with $U_{\star} E_{\star}=S_{1}$. Since $S_{1}$-Alg differs from $S_{*}-$ Alg it follows that $U_{*}$ is not tripleable.

Clearly, all operational categories are $S_{*}$-algebras and there is a commuting triangle

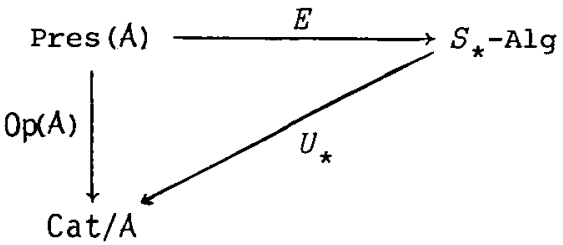

In turn, it will be shown that $S_{*}$-algebras "are" operational categories by constructing a right inverse for $E$. The presentations thus constructed will all employ the same theory, $\theta_{0}$.

3. The characterisation theorem

DEFINITION 3.1. The standard theory $\theta_{0}: B_{0} \rightarrow T_{0}$ is given as follows: $B_{0}$ is freely generated by the graph

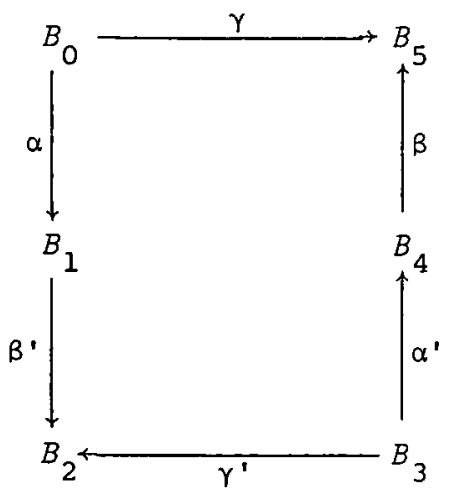

and $T_{0}$ is generated by 


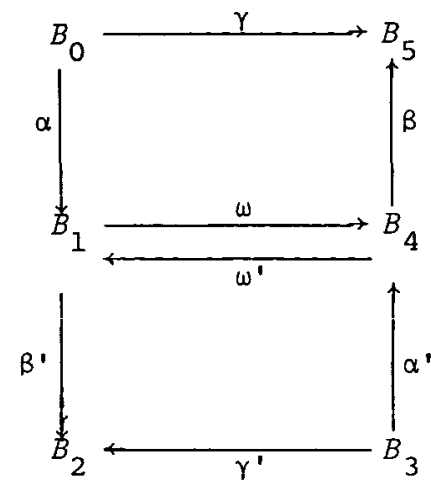

and the equations

$$
\begin{aligned}
\beta \omega \alpha & =\gamma \\
\beta^{\prime} \omega^{\prime} \alpha^{\prime} & =\gamma^{\prime} \\
\omega^{\prime} \omega & =1 \\
\omega \omega^{\prime} & =1 .
\end{aligned}
$$

$\theta_{0}$ is the inclusion. (See [2] for a construction of a category from a sketch). $\operatorname{Pres}_{0}(A)$ is the subcategory of Pres (A) with the objects of the form $\left(\theta_{0}, H\right)$ and morphisms $(j, k)$ where $j$ is the identity.

THEOREM 3.2. There is a right inverse $M$ for $E$ factorising through $\operatorname{Pres}_{0}(\mathrm{~A})$ such that $O p(\mathrm{~A}) . M=U_{\star}$. Consequently, the following are equivalent:

(i) $U: D \rightarrow A$ is operational,

(ii) $U: D \rightarrow A$ underities an $S_{*}$-algebra,

(iii) $U: D \rightarrow A$ is operational with respect to $\theta_{0}$.

Proof. For each $S_{\star}$-algebra a base functor will be constructed. Other details may be found in [6]. Firstly, for any $U: D \rightarrow A$ in Cat/A, define $C D$ by the pushout (in Cat)

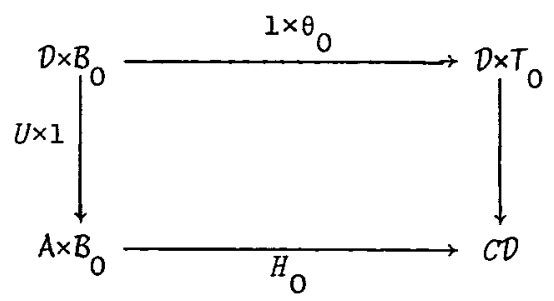


(Note in passing that $0 \mathrm{p}_{0}(A)$ has a left adjoint, whose value at $D$ is $\left(\theta_{0}, H_{0}\right)$, though $O \mathrm{p}(\mathrm{A})$ never does.)

$C D$ may be generated by the following sketch: each object $A$ in $A$ yields a copy of $B_{O}$ in $A \times B_{O}$ whose image in $C D$ is written

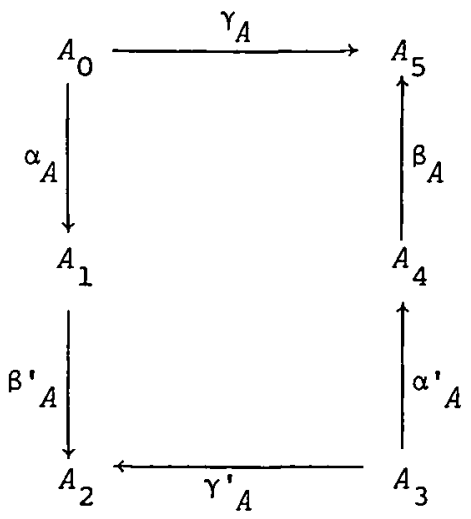

Since $I \times \theta$ is bijective on objects, $H_{0}$ is and so all objects of $C D$ are of the form $A_{i}$. Each object $D$ of $D$ yields a pair of inverses $\omega$ and $\omega^{\prime}$ in $C D$ pictured as follows:

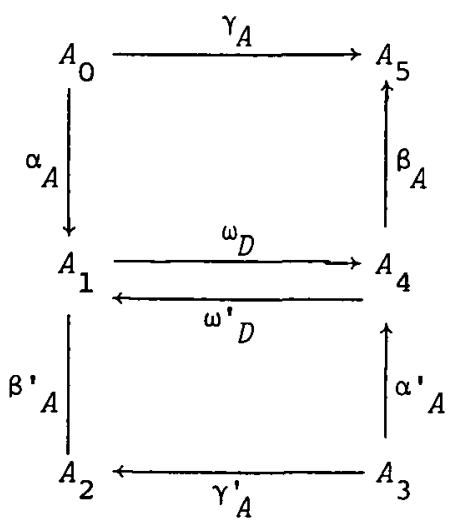

where $A=U D$. They also satisfy the equations

$$
\begin{aligned}
{ }_{A}{ }^{\omega} D_{A}{ }_{A} & =\gamma_{A} \\
\beta_{A}^{\prime} \omega_{D}^{\prime} \alpha_{A}^{\prime} & =\gamma_{A}^{\prime} .
\end{aligned}
$$

Given $f: A \rightarrow A^{\prime}$ in $A$ we have $f_{i}=H_{0}\left(f, B_{i}\right): A_{i} \rightarrow A_{i}^{\prime}$ for each $i$. 
The $f_{i}^{\prime} s$ compose as they do in $A$ and commute with the morphisms from $B_{0}$, for example $f_{1} \alpha_{A}=\alpha_{A}, f_{0}$. Given $f: D \rightarrow D^{\prime}$ in $D$ then $U f$ has the additional property that $(U f)_{4} \omega_{D}=\omega_{D^{\prime}}(U f)_{1}$ and similarly for $\omega^{\prime}$. $S_{1} D$ is given by the pullback

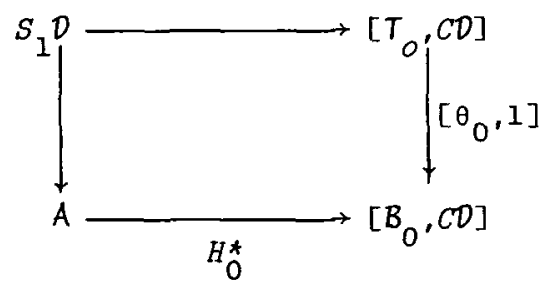

Now when $\left(D,\left\{d_{n}\right\}\right)$ is an $S_{*}$-algebra we define $q_{1}: C D \rightarrow C_{1} D$ to be the quotient of $C D$ given by imposing the relations

$$
\begin{aligned}
& y_{4}{ }^{\omega} D^{x_{1}}=\omega_{D^{\prime}} \\
& y_{1}{ }^{\prime}{ }^{\prime} x_{4}=\omega^{\prime}{ }^{\prime} .
\end{aligned}
$$

whenever $D^{\prime}=d_{n}(y, D, x)$ for some $n$-shuffle retract. Then

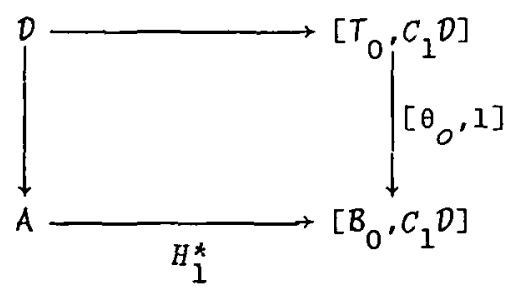

is a pullback (where $H_{1}=q_{1} H_{0}$ ) as required.

Note that $M$ is not left adjoint to $E$ and $S_{*}$-Alg is not equivalent to Pres $_{0}(A)$.

\section{Props}

We may ask that our presentations have some extra structure, say, a monoidal structure or that of finite products, and perform our constructions, not in Cat, but in some other appropriate category, say, the category of symmetric monoidal categories and strong symmetric monoidal functors, denoted StM. Below we examine the monoidal case 
(see [5] for terminology), in which props (MacLane [11]) become examples of theories. In this paper all monoidal categories and functors are assumed symmetric.

Denote by $\operatorname{StM}(B, C)$ the monoidal category of strong monoidal functors from $B$ to $C$ and let $(\overline{)}$ : Cat $\rightarrow$ Stm be the left bi-adjoint ([13]) to the forgetful functor, with unit $\eta: \mathcal{C} \rightarrow \overline{\mathcal{C}}$.

A monoidal presentation $(\theta, H)$ consists of a prop $\theta$, by which is meant a strong monoidal functor $\theta=\left(\theta, \tilde{\theta}, \theta^{0}\right): B \rightarrow T$ in which $\theta$ is bijective on objects, and a (strong monoidal) functor $H=\left(h, \tilde{h}, h^{0}\right): A \rightarrow \operatorname{Stm}(B, C)$.

PROPOSITION 4.1. Let $(\theta, H)$ be a monoidal presentation. Then there is a puzzback

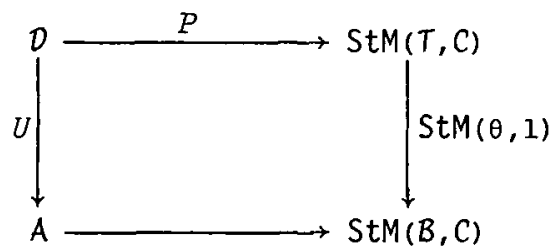

in StM and $U=\left(u, u, u^{O}\right): D \rightarrow A$ is strict.

Proof. It is well known that StM has all pseudo-limits (as defined in [7]). The pullback $u: D \rightarrow A$ of $\operatorname{StM}(\theta, 1)$ by $h$ in Cat is equivalent to a subcategory of the pseudo-pullback determined by the presentation. The result follows.

A strict monoidal functor $U: D \rightarrow A$ arising from a monoidal presentation as above is called a category of prop algebras. Any category equivalent to such a category of algebras is called proppable.

EXAMPLE 4.2. Let $P$ be a skeleton of $\bar{I}$. Its objects may be identified with the natural numbers and

$$
P(m, n)= \begin{cases}\phi & m \neq n \\ P_{n} & m=n\end{cases}
$$

where $P_{n}$ is the permutation monoid on $n$ elements. On objects the 
tensor is given by addition. Let $\theta: P \rightarrow T$ be a strict prop. Then $T$ is a prop in MacLane's sense $([11])$. Let $A$ be a monoidal category. Define $H=\left(h, \tilde{h}, h^{0}\right): A \rightarrow \operatorname{StM}(P, A)$ by $(h A) n=A^{n}=A \otimes A \otimes \ldots \otimes A$ ( $n$ times) and $(h A) \sigma$ is the composite of symmetries which permutes the $A$ 's in (hA)n according to $\sigma$, together with the obvious choices of $\tilde{h}$ and $h^{\circ}$. Then $D=\operatorname{StM}[T, A]$ is proppable in Barr's sense ([1]). Note that the following square (whose horizontal maps are the inclusions)

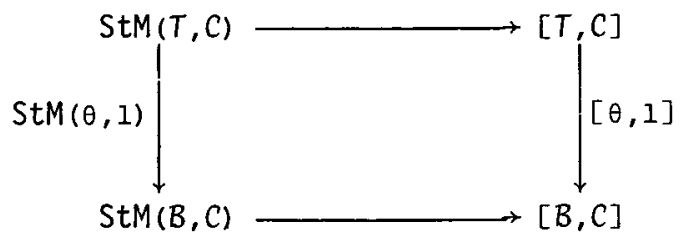

is not a pullback. Thus, it is not immediate that proppable categories are operational.

THEOREM 4.3. The folzowing are equivalent:

(i) $U: D \rightarrow A$ is proppable,

(ii) $U$ is strict monoidal and operational,

(iii) $U$ is proppable with respect to $\bar{\theta}_{0}$.

Proof. (iii) $\Rightarrow$ (i) is trivial.

(i) $\Rightarrow$ (ii): $U$ is strict monoidal by Proposition 4.1 and straightforward verification shows that $D$ is an $S_{*}$-algebra. Now use Theorem 3.2 .

(ii) $\Rightarrow$ (iii): Without loss of generality assume that $D$ is a category of algebras with presentation $\left(\theta_{0}, H_{0}\right)$ as in the proof of (ii) $\Rightarrow$ (iii) in Theorem 3.2. $H_{0}$ does not underly a monoidal functor. This problem is overcome by choosing a $C_{2} D$ and $Q_{2}=\left(q_{2}, \tilde{q}_{2}, q_{2}^{o}\right): C_{1} D \rightarrow C_{2} D$ with the following property: there is a strong monoidal functor $H_{2}=\left(h_{2}, \tilde{h}_{2}, h_{2}^{0}\right)$ such that $h_{2}=q H_{0}$ and for any monoidal category $V, \operatorname{StM}\left(C_{2} D, V\right)$ is equivalent to the full subcategory of $\operatorname{StM}\left(C_{1} D, V\right)$ of monoidal functors $R=\left(r, \tilde{r}, r^{O}\right)$ such that $r H_{0}$ 
underlies a strong monoidal functor. Then, as in Theorem 3.2, we have the pullback

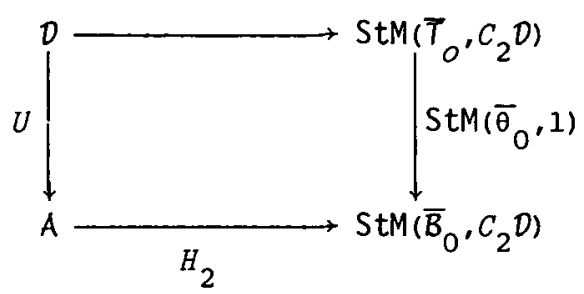

showing that $D$ is proppable over $A$.

\section{References}

[1] Michael Barr, "Coalgebras over a commutative ring", J. Algebra 32 (1974), 600-610.

[2] M. Barr and C. Wells, Toposes, Triples and Theories,

(Springer-Verlag, New York, Berlin, Heidelberg, Tokyo, 1985).

[3] Robert Davis, "Equational systems of functors", Reports of the

Micwest Category Seminar 1965, 92-109 (Lecture Notes in

Mathematics 47. Springer-Verlag, Berlin, Heidelberg,

New York, 1967).

[4] Yves Diers, "Foncteur pleinement fidele dense classant les algebres", Cahiers Topologie Geom. Differentielle 17 (1976), 171-186.

[5] Samuel Eilenberg and G. Max Kelly, "Closed Categories", Proceedings of the Conference on Categorical Algebra La Jolla (1965), 421-562 (Springer-Verlag, Berlin, Heidelberg, New York, 1966).

[6] C. Barry Jay, Generalising the Structure-Semantics Adjunction: Operational Categories (thesis, MCGill University, 1984).

[7] G.M. Kelly and Ross Street, "Review of the elements of 2-categories", Category Seminar Sydney 1972/73, 75-103, (Lecture Notes in Mathematics, 420, Springer-Verlag, Berlin, Heidelberg, New York, 1974). 
[8] F.W. Lawvere, Functorial semantics of algebraic theories (thesis, Columbia University, 1963).

[9] F.E.J. Linton, "Some aspects of equational systems", Proceedings of the Conference on Categorical Algebra La Jolla 1965, 84-94, (Springer-Verlag, Berlin, Heidelberg, New York, 1966).

[10] F.E.J. Linton, "nn outline of functorial semantics", Seminar on Triples and Categorical Homology Theory E.T.A. Zurich 1966/67, 7-52 (Springer-Verlag, Berlin, Heidelberg, New York, 1969).

[11] Saunders MacLane, "Categorical algebra", BuZZ. Amer. Math. Soc. 71 (1965), 40-106.

[12] Saunders MacLane, Categories for the working mathematician (Springer-Verlag, New York, Heidelberg, Berlin, 1971).

[13] Ross Street, "Fibrations in bicategories", Cahiers Topolgie Geom. Differentieile 21 (1980), 111-160.

[14] Michel Thiébaud, Self-dual structure-semontics and algebraic categories (thesis, Dalhousie University, 1971).

[15] Oswald Wyler, "Operational categories", Proceedings of the Conference on Categorical Algebra La Jolla 1965, 295-316, (Springer-Verlag, Berlin, Heidelberg, New York, 1966).

Department of Pure Mathematics,

University of Sydney,

N.S.W. 2006,

Australia. 